\title{
Some questions on Cohen-Macaulay rings
}

\author{
By \\ Masayoshi Nagata
}

(Received, July 21, 1972; Revised Nov. 27, 1972)

In the present paper, we shall deal with the following type of problem on Cohen-Macaulay rings.

Let $R=\sum_{i=0}^{\infty} R_{i}$ be a graded noetherian ring, $R_{i}$ being the module of homogeneous elements of degree $i$. If $R_{0}$ is a field, then it is easy to see that $R$ is Cohen-Macaulay if and only if $R_{M}$ is CohenMacaulay, $M$ being the irrelevant prime ideal (i. e., $M=\sum_{i \geq 1} R_{i}$ ).

Our problem is to generalize this characterization.

For instance, one can ask the following questions:

Question 1. Assume that $R_{M}$ is Cohen-Macaulay for every maximal ideal $M$ such that $M \supseteq \sum_{i \geq 1} R_{i}$. Does it follow that $R$ is Cohen-Macaulay?

Question 2. Let $K$ be the total quotient ring of $R_{0}$. Then, does the condition that both $R \bigotimes_{R_{0}} K$ and $R_{0}$ are Cohen-Macaulay imply that $R$ is Cohen-Macaulay ?

To the writer's knowledge, Question 1 is unsolved yet and Question 2 has a negative answer. In the present paper we discuss some facts related to these questions, and main results relate to the case where $R$ is a projective module over $R_{0}$.

In connection with these question we ask the following

Question 3. Assume that $R$ is Cohen-Macaulay. Does it follow 
that $R_{0}$ is Cohen-Macaulay?

We have really a negative answer to this question, and on the other hand, we have an affirmative answer under an additional assumption that $R$ is a projective $R_{0}$-module.

We add here a remark that the condition that $R$ is a projective $R_{0}$-module is equivalent to that $R$ is a flat $R_{0}$-module. This fact follows from that each $R_{i}$ is a finite $R_{0}$-module (for, $R$ is noetherian and therefore $R_{0}$ is noetherian and $R$ is finitely generated over $R_{0}$ ).

As for the term "Cohen-Macaulay ring", we understand it as the same as "locally Macaulay ring", namely, it is a noetherian ring $T$ such that for every maximal ideal $M$ of $T$, the local ring $T_{M}$ has a system of parameters which is a regular sequence.

Throughout this article, we maintain the notation that $R=\sum_{i=0}^{\infty} R_{i}$ is a graded noetherian ring, $R_{i}$ being the module of homogeneous elements of degree $i$.

The writer wishes to express his thanks to Professor Lascu of the Univercity of Montreal pointing out a wrong statement contained in Theorem 1.1 in the first manuscript of the present article.

\section{The case where $R_{0}$ is an Artin ring.}

Theorem 1. 1. Assume that $R_{0}$ is an Artin ring. If $R_{M}$ is Cohen-Macaulay for every maximal ideal $M$ containing $\sum_{i \geq 1} R_{i}$, then $R$ is a Cohen-Macaulay ring.

Proof. $\quad R_{0}$ is a direct sum of Artin local rings: $R_{0}=R_{1}^{\prime} \oplus \cdots \oplus R_{\varsigma}^{\prime}$ Let $e_{i}$ be the identity of $R_{1}^{\prime}$. Then $R$ is the direct sum of $R e_{i}$ $(i=1, \cdots, s) ; M$ contains all $e_{j}$ except for only one $e_{i}$ and $R_{M}=$ $\left(R e_{i}\right)_{M e_{i}}$. Therefore, it is sufficient to prove the assertion under the assumption that $R_{0}$ is an Artin local ring. Let $m$ be the maximal ideal of $R_{0}$, then $M=m+\sum_{i \geq 1} R$. Now, if $R_{0} / m$ contains only a finite number of elements, we consider $R_{0}(x)$ instead of $R_{0}$.

Thus we can reduce to the case where $R_{0} / m$ contains infinitely 
many elements. Since $R$ is noetherian, $R$ is finitely generated over $R_{0}$. Therefore, there is an $R_{i}$, say $R_{d}$ such that $R$ is integral over $R_{0}\left[R_{d}\right]$. Then, by the normarization theorem, there are $z_{1}, \cdots, z_{t} \in R_{d}$ such that (i) $z_{1}, \cdots, z_{t}$ are algebraically independent over $R_{0}$ and (ii) $R_{0}\left[R_{d}\right]$ is integral over $F=R_{0}\left[z_{1}, \cdots, z_{t}\right]$. Then $R$ is integral over $F$. Then $M \cap F=m+\sum z_{i} F$. Since $R_{M}$ is Cohen-Macaulay and since $z_{1}, \cdots, z_{t}$ is a system of parameters of $R_{M}$, we see that length $R_{M} /\left(z_{1}, \cdots, z_{t}\right)^{n}=$

(length $R_{M} /\left(z_{1}, \cdots, z_{t}\right)$ ) (length $\left(R_{0} / m\right)\left[z_{1}, \cdots, z_{t}\right] /\left(z_{1}, \cdots, z_{t}\right)^{n}$ ).

Therefore the theorem of transition holds for the extension $R_{M} / R_{0}\left[z_{1}, \cdots, z_{t}\right]_{\left(z_{1}, \cdots, z_{t}, m\right) \text {. }}$

Let $f_{1}, \cdots, f_{n}$ be a set of homogeneous generators for $R$ over $R_{0}\left[z_{1}, \cdots, z_{t}\right]$ and let $N$ be the kernel of the natural surjection of a free module $F=\sum_{i=1}^{u_{1}} R_{0}\left[z_{1}, \cdots, z_{t}\right] X_{i} \rightarrow R$. Let $\left\{g_{i j}(j=1, \cdots, n(i)\right.$; $i=1, \cdots, e$ with $e$ such that $\left.\left.m^{e}=0\right)\right\}$ be a set of homogeneous elements of $N$ (with a gradation defined by $\operatorname{deg} X_{i}=\operatorname{deg} f_{i}$ ) such that $g_{i 1}, \cdots, g_{i, n(i)}$ modulo $m^{i} N+\sum z_{i} F$ form a linearly independent basis for $m^{i-1} N /\left(m^{i} N+\left(\sum z_{j} F \cap N\right)\right)$ over the field $R_{0} / m$. Then, letting $\left\{U_{i j}(j=1, \cdots, h(i) ; i=1,2, \cdots)\right\}$ be the set of all monomials in $z_{1}, \cdots, z_{t}$ with $\operatorname{deg} U_{i j}=i$, we see that $G_{i}=\left\{g_{i j} U_{\alpha \beta}\right.$ (for all possible $\left.\left.j, \alpha, \beta\right)\right\}$ form a linearly independent basis for $m^{i-1} N / m^{i} N$ over the field $R_{0} / m$.

This property and the fact that $R_{0}\left[z_{1}, \cdots, z_{t}\right]$ is a Cohen-Macaulay ring imply that $R$ is Cohen-Macaulay.

\section{Question 3.}

We begin with an affirmative case:

Theorem 2.1. Assume that $R$ is a projective module over $R_{0}$ and that $R_{M}$ is Cohen-Macaulay for every maximal ideal $M$ containing $\sum_{i \geq 1} R_{i}$. Then $R_{0}$ is Cohen-Macaulay.

Proof. We may assume that $M \cap R_{0}=m_{0}$ is the unique maximal ideal of $R_{0}$. If $m_{0}$ contains a non-zero-divisor $a$, then considering $R / a R$, we can proceed with our proof by induction on Krull $\operatorname{dim} R_{0}$. 
Therefore we assume that $m_{0}$ consists only of zero-divisors. Let $x_{1}, \cdots, x_{n}$ be homogeneous elements of $R$ which generate $R$ over $R_{0}$. We consider a polynomial ring $P=R_{0}\left[X_{1}, \cdots, X_{n}\right]$ with a gradation such that $\operatorname{deg} X_{i}=\operatorname{deg} X_{i}$. Then we have an $R_{0}$-homomorphism $\varphi$ : $P \rightarrow R$ so that $\varphi X_{i}=x_{i}$. Let $I$ be the kernel $\varphi$. I is homogeneous, and we have an irredundant expression $I=Q_{1} \cap \cdots \cap Q_{s}$ with homogeneous primary ideals $Q_{1}, \cdots, Q_{s}$. Now, our assumption that $m_{0}$ consists only of zero-divisors means that some of these $Q_{i}$, say $Q_{1}$, $\cdots, Q_{t}(t \geq 1)$ contain some power of $m_{0}$. If all of these $Q_{i}$ contain a power of $m_{0}$, then $m_{0}$ must be nilpotent and $R_{0}$ is an Artin ring, Assume the contrary, i. e., $\sqrt{Q_{i}} \nsupseteq m_{0}$ for $i=t+1, \cdots, s ; t<s$. Since $R=P / I$ is Cohen-Macaulay, $I$ has no imbedded prime divisor. Therefore, for each $i>t$, there is a homogeneous element $y_{i} \in Q_{i}$ which is not in any $\sqrt{Q_{1}}, \cdots, \sqrt{Q_{t}}$. Note that deg $y_{i}>0$ because $\sqrt{Q_{j}} \supseteq m_{0}$ for every $j \leq t$. Set $y=y_{t+1} \cdots y_{s}$ and let $m^{\prime \prime}$ be a power of $m_{0}$ such that $m^{\prime \prime} \subseteq \bigcap_{j \leq t} Q_{j}$. Then $m^{\prime \prime} y \subseteq I$. Since $R$ is a free $R_{0}$-module and since $m^{\prime \prime} \neq 0$, we see that $y \in \operatorname{ker} \varphi=I$, which contradicts our assumption that $y_{i} \notin \sqrt{Q_{j}}$ for $j \leq t$.

Next we give an example which shows that Question 3 has a negative answer.

Let $\left(R_{0}, m_{0}\right)$ be a (noetherian) local ring of Krull dimension one such that $m_{0}$ consists only of zero-divisors. Then

$$
0=q_{1} \cap \cdots \cap q_{u}
$$

with primary ideals $q_{1}, \cdots, q_{u}$ such that $\sqrt{q_{1}}=m_{0}$ and $\sqrt{q_{i}} \neq m_{0}$ for every $i \geq 2$. Consider a polynomial ring $P=R_{0}[X]$ whih gradation such that $\operatorname{deg} X=1$. Set $Q_{i}=q_{i} P+X P$ for $i \geq 2$ and $Q_{1}=q_{1} P$. Then

Example 2. 2. $R=P /\left(Q_{1} \cap \cdots \cap Q_{u}\right)$ is the required example.

Proof. Each $Q_{i}$ is obviously a primary ideal of $P$, and Krull $\operatorname{dim} P / Q_{i}=1$. Therefore $R$ is of Krull dimension one, and the zero ideal of $R$ has no imbedded prime divisor. Thus $R$ is CohenMacaulay. $\quad Q_{i} \cap R_{0}=q_{i}$ and therefore $R_{0} \cap Q_{1} \cap \cdots \cap Q_{u}=0$. Therefore 
under the natural gradation, the degree zero part of $R$ is $R_{0}$ which is not Cohen-Macaulay because $m_{0}$ consists only of zero-divisors.

We add here one obvious remark: It is not true that " $R$ is Cohen-Macaulay $\Rightarrow R$ is a projective $R_{0}$-module". The example above is one of such examples. There are many other much easier examples to this. For instance, we get such an example among $R=R_{0}\left[x_{1}, \cdots\right.$, $\left.x_{n}\right] /(l)$ with $\left(R_{0}, m\right)$ a Cohen-Macaulay local ring of Krull $\operatorname{dim} \geq 1$ and with $l=a_{1} x_{1}+\cdots+a_{n} x_{n} ; a_{i} \in m$.

Therefore, for further investigation of these questions, it is necessary to find out a good method to deal with the case where $\left(R_{0}, m\right)$ is Cohen-Macaulay local ring of Krull $\operatorname{dim} \geq 1$ and every elements of $m$ is a zero divisor in $R$.

\section{Questions 1,2 in case $R$ is a projective $R_{0}$-module.}

Theorem 3.1. Assume that $R$ is a projective module over $R_{0}$. If $R_{M}$ is Cohen-Macaulay for every maximal ideal $M$ containing $\sum_{i \geq 1} R_{i}$, then $R$ is Cohen-Macaulay.

Proof. Let $d=$ Krull $\operatorname{dim} R_{0}$. Let $N$ be an arbitrary maximal ideal of $R$ and we have only to show that $R_{N}$ is Cohen-Macaulay. Let $S=R_{0}-(N \cap R)$. Then considering $R_{S}$ instead of $R$, we may assume that $R_{0}$ is a local ring and that $m=N \cap R_{0}$ is maximal. If $d=$ height $m=0$, then $R$ is Cohen-Macaulay by Theorem 1.1. If $d \geq 1$, then let $a_{1}, \cdots, a_{d}$ be a system of parameters of $R_{0}$. By virtue of Theorem 2.1, $R_{0}$ is Cohen-Macaulay, hence $a_{1}$ is not a zero-divisor in $R_{0}$. Since $R$ is a projective module over the local ring $R_{0}, a_{1}$ is not a zero-divisor in $R . R / a_{1} R$ is a projective module over $R_{0} / a_{1} R_{0}$, and therefore by an induction on $d$, we see that $R / a_{1} R$ is CohenMacaulay. Since $a_{1}$ is not a zero-divisor, It follows that $R_{N}$ is Cohen-Macaulay.

q.e.d.

It is nearly obvious that Question 2 has a negative answer. But we give hare a concrete example.

Let $\left(R_{0}, m_{0}\right)$ be a Cohen-Macaulay local integral demain of Krull 
dimension at least one. Let $P$ be the polynomial $\operatorname{ring} R_{0}\left[X_{1}, \cdots, X_{n}\right]$ with $n \geq 2$. Let $Q$ be the prime ideal of $P$ generated by $X_{1}, \cdots, X_{r}$, where the integer $r$ is chosen so that $r \geq 1$ and that $r \neq$ Krull dim $R_{0}$. Let $Q^{\prime}$ be the prime ideal $m_{0} P$. Considering the natural gradation, we have

Example 3. 2. $R=P /\left(Q \cap Q^{\prime}\right)$ is the required example.

Proof. Since $Q \cap R_{0}=0$, we see that the degree zero part of $R$ is $R_{0}$ and is Cohen-Macaulay by our assumption. Since Krull $\operatorname{dim} P / Q^{\prime}$ $=n$ and since Krull $\operatorname{dim} P / Q=n-r+$ Krull $\operatorname{dim} R_{0} \neq n$, we see that $R$ is not Cohen-Macaulay. Let $K$ be the field of quotients of $R_{0}$. Then $R \otimes K \cong(P \otimes K) /\left(Q \otimes K \cap Q^{\prime} \otimes K\right)$. Since $Q^{\prime} \supseteq m_{0}$, we see that $Q^{\prime} \otimes K \ni 1$, whence $R \otimes K \cong K\left[X_{1}, \cdots, X_{n}\right] /\left(X_{1}, \cdots, X_{r}\right) \cong K\left[X_{r+1}, \cdots, X_{n}\right]$,

Even if we add an assumption that $R$ is a projective module over $R_{0}$, we still have a negative answer of Question 2 .

Example 3. 3. Let $R_{0}$ be a discrete valuation ring with $a$ prime element $p$. Let $u, v$ be algebraically independent elements over $R_{0}$. Let $R$ be $R_{0}\left[u^{3}, p u^{2} v, u v^{2}, v^{3}\right]$ with $\operatorname{deg} u=\operatorname{deg} v=1 / 3$. Then $R$ is the required example.

Proof. Consider the natural homomorphirm $\varphi: R_{0}[x, p, z, w] \rightarrow R$. The kernel $I$ of $\varphi$ is generated by $y^{2}-p^{2} z x, y^{3}-p^{3} w x^{2}, z^{3}-w^{2} x, y z-$ $p w x, y w-p z^{2}$. Hence $\varphi_{p}:\left(R_{0} / p R_{0}\right)[x, y, z, w] \rightarrow R / p R$ has kernel $I_{p}$ generated by $y^{2}, y^{3}, y z, y w, z^{3}-w^{2} x$. Therefore $p, x$ form a maximal $R$-sequence in the homogeneous maximal ideal, hence $R$ is not CohenMacaulay. On the other hand, $R \otimes K$ is nothing but $K\left[u^{3}, u^{2} v, u v^{2}\right.$, $\left.v^{3}\right]$, which is clearly Cohen-Macaulay. Since $R_{0}$ is a discrete valuation ring, we see easilly that $R$ is $R_{0}$-free.

q. e. d.

KYOTO UNIVERSITY 